\title{
SEGMENTING EXTENDED STRUCTURES IN RADIO ASTRONOMICAL IMAGES BY FILTERING BRIGHT COMPACT SOURCES AND USING WAVELETS DECOMPOSITION
}

\author{
Institut d'Informàtica i Aplicacions \\ Universitat de Girona \\ 17071, Girona, Spain \\ martapb@eia.udg.edu
}

Marta Peracaula, Arnau Oliver, Albert Torrent, Xavier Lladó, Jordi Freixenet and Joan Martí

\begin{abstract}
The automatic segmentation of extended real structures (such as SNRs, HII regions, bow shocks, etc) in large surveys is a difficult task due to their morphological complexity and their wide variety in scale and surface brightness. With the aim of dealing with these issues we propose in this paper an automatic segmentation method based on applying wavelet decomposition in the residual thresholded images. The use of this strategy, instead of a wavelet decomposition on the original image, allows to avoid the artifacts produced by strong sources. Experimental results with radio images demonstrate that the proposed method successfully segments extended structures at different scales and therefore is suitable for further morphological analysis and object recognition processes.
\end{abstract}

Index Terms - Astronomical image analysis, segmentation, wavelet decomposition.

\section{INTRODUCTION}

Sky surveys in the low frequency ranges of the electromagnetic spectrum reveal thousands of emitting objects shown at different intensity scales. When imaging rich areas in the interstellar medium, many of the compact sources overlap with objects associated to extended, morphologically complex real structures, such as Supernova Remnants (SNRs), HII regions and bow shocks. The wide variety in spacial scale and surface brightness of these sources make their automatic detection and segmentation a difficult task. To illustrate these facts, we use the radioimage corresponding to the high galactic longitude end of the Phase I Canadian Galactic Plane Survey (CGPS hereafter) [1]. Figure 1 shows, on the top, the image composition corresponding to mosaics V1, V2, W1, W2, $\mathrm{X} 1, \mathrm{X} 2$, Y1 and Y2 of the CGPS $1420 \mathrm{MHz}$, continuum. We have eliminated $0.1 \%$ of the intensity outliers in order to visualize some dozens of sources. Nevertheless, the data corresponding to these mosaics contain thousands of objects, as

The authors acknowledge support the Spanish Ministerio de Educación y Ciencia (MEC) under grant AYA2010-21782-C03-02, as well as partial support by the European Regional Development Fund (ERDF/ FEDER). it is illustrated when we display at different intensity scales the sub-image contained in the red square. The bottom left of the figure shows the sub-image with $2 \%$ of the outliers eliminated, whereas in the right $5 \%$ of the outliers are removed. On top of a great number of compact sources, several extended structures and its surrounding emission appear (the zoomed area contains Lynds Bright Nebula 679).

In previous works we focused our attention on the development of new techniques suitable for the detection of faint compact objects [2, 3]. However, in this paper we focus on the automatic segmentation of extended and irregular structures in low frequency astronomical images for further catalogation and morphological analysis. In the context of segmentation, wavelet image decomposition has been proved as a powerful tool to detect and separate objects that can be represented at different spatial frequencies [4]. However, the high intensity dynamic range of these kind of images diminishes the performance of the decomposition. To avoid this problem a new strategy is presented in this paper. The rest of the paper is structured as follows: next section describes our approach for detecting extended faint structures in low frequency astronomical surveys, while the results are presented in Section 3. The paper ends with conclusions.

\section{SEGMENTING EXTENDED STRUCTURES BY USING THE “À TROUS” ALGORITHM}

Figure 2 shows a graphical representation of the proposed algorithm using a sample image. We include on the bottom left part of the figure a contrast stretched sample image in order to be able to compare it with the obtained segmentation result. Our approach mainly consists in three different steps. Firstly, the brightest regions of the image are detected and removed (note that these regions correspond mainly to compact sources). Secondly, a wavelet decomposition is used to permit a multi-resolution analysis. Finally, extended regions are found as the addition of the automatic thresholding of the last two planes of the decomposition.

In this section we will provide the details of our approach, 


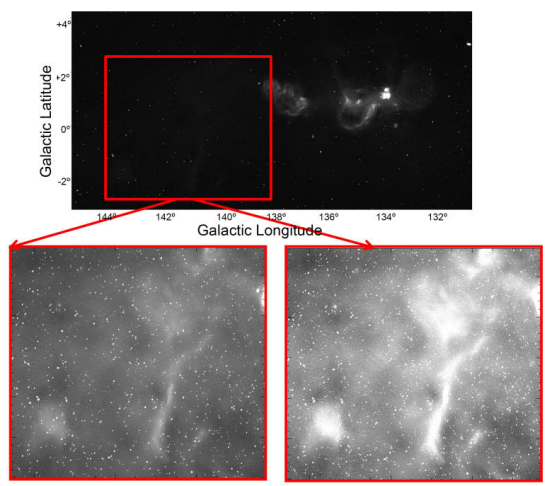

Fig. 1. Top: Contrast stretched ( $0.1 \%$ outliers eliminated $)$ image corresponding to mosaics V1, V2, W1, W2, X1, X2, Y1 and Y2 of Phase I CGPS at $1420 \mathrm{MHz}$, continuum. Bottom: Zoomed area around Lynds Bright Nebula 679, displayed with $2 \%$ of outliers eliminated (left) and $5 \%$ of outliers eliminated (right)

presenting firstly the wavelet decomposition used and then the proposal for the segmentation of extended structures in low frequency images.

\subsection{Wavelet decomposition using the "à trous" algorithm}

Multiscale Vision Models [5] decompose an image in $J$ scales or wavelet planes and segment independently each of the images representing a scale. Since astronomical sources are mostly isotropic (stars, clusters, galaxies, etc) researchers generally choose to use a wavelet transform that does not privilege any direction in the image and maintains also the sampling at each scale [6]. For this reason one of the widely used transforms in this field is the Stationary Wavelet Transform (SWT) also called "à Trous" algorithm.

In the common wavelet transform (DWT) the results at each level are half the size of the original sequence. In contrast, using the SWT the signal is never sub-sampled. Instead, the filters have to be up-sampled at each level of decomposition, by padding the corresponding low-pass and high-pass filters with zeros. Physically, the major advantages of SWT over DWT is the preservation of spatial information of the original image at each level, achieving the translation invariance which is not possible in the traditional DWT.

Mathematically, the SWT is a decomposition of an image $I(k, l)$ in $J$ scales or wavelet planes $W_{j}(k, l)$ and a smoothed array $F_{J}(k, l)$ using a smoothing filter $h$ (associated to the wavelet scaling function) and is defined in the following way:

$$
I(k, l)=F_{J}(k, l)+\sum_{j=1}^{J} W_{j}(k, l)
$$

$F_{J}(k, l)$ and $W_{j}(k, l)$ are calculated through the following iterative process:

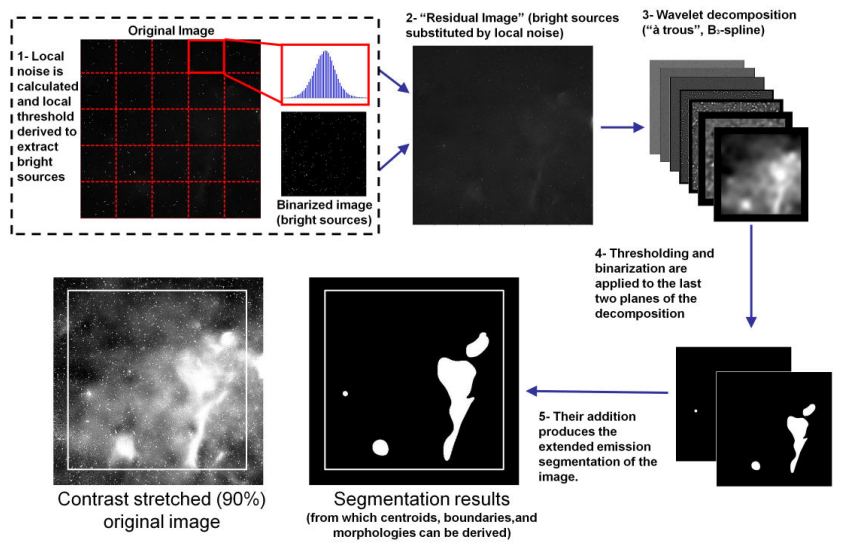

Fig. 2. Graphical representation of the proposed algorithm and visual comparison of the segmentation result with the contrast stretched image.

$$
\begin{aligned}
F_{0}(k, l) & =I(k, l) \\
F_{j}(k . l) & =\left\langle H_{j}, F_{j-1}\right\rangle(k, l) \\
W_{j}(k, l) & =F_{j-1}(k, l)-F_{j}(k, l)
\end{aligned}
$$

with $j=1, \ldots, J$ and

$$
\left\langle H_{j}, F_{j-1}\right\rangle(k, l) \equiv \sum_{n, m} h(n, m) F_{j-1}\left(k+2^{j-1} n, l+2^{j-1} m\right)
$$

The set $W_{1}, W_{2}, \ldots, W_{J}, F_{J}$ represents the wavelet transform of the data.

Following Starck \& Murtagh [7] and references therein, in this work we will use as scaling function the $B_{3}$-spline function, which is very similar to a Gaussian one. In this way the mask associated to the filter $h$ takes the following form:

$$
h \equiv \frac{1}{256}\left(\begin{array}{ccccc}
1 & 4 & 6 & 4 & 1 \\
4 & 16 & 24 & 16 & 4 \\
6 & 24 & 36 & 24 & 6 \\
4 & 16 & 24 & 16 & 4 \\
1 & 4 & 6 & 4 & 1
\end{array}\right)
$$

As an example of this transformation, Figure 3 shows an image containing mosaics V1, V2, W1 and W2 of the CGPS at $1420 \mathrm{MHz}$ (continuum), and its decomposition in 6 scales plus the smoothed array using the "à trous" algorithm. As it can be seen, low index scales emphasize high spatial frequencies (which corresponds to compact and semi-compact objects in case of true signal), while high index scales emphasize low spatial frequencies (in this case extended source structures).

\subsection{Detection of extended structures}

In order to detect extended structures we use the SWT. However, as it is shown in Figure 4 the direct application of this transformation has two main issues: 


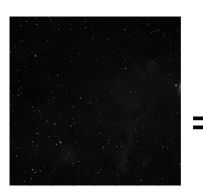

I

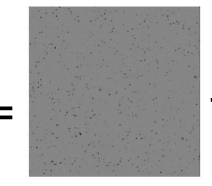

$W 1$

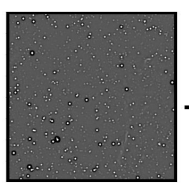

W4

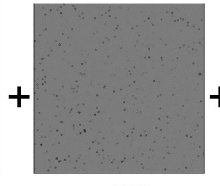

$W 2$

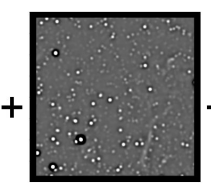

W5

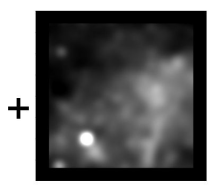

F6

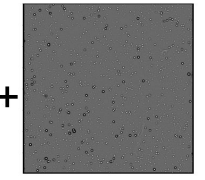

W3

W6

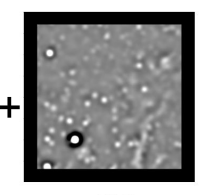

Fig. 3. Multi-resolution wavelet decomposition of a sample image. From top left to bottom right going from higher to lower spatial frequencies. Note that the segmentation of extended structures has to be done at low spatial frequencies.

- Strong compact sources are not filtered out and show up in low spatial frequency planes. This is due to the high brightness of those structures.

- Negative artifacts around strong compact source structures appear and pollute. This is due to the necessity of keeping the wavelet coefficient mean at zero.

Hence, in order to detect the extended structures we propose to create a novel image where bright sources are replaced by local noise. Subsequently, the wavelet decomposition is applied to this new image in order to avoid the polluting effects of strong sources. It is commonly assumed that noise in radio-astronomical images follows a Gaussian distribution [6]. Hence, bright sources are found as the brighter outliers of the local Gaussian distribution fitting of the original image subregions (thresholding process). After their detection, we ensure that these objects are not affecting further analysis by enlarging them using the morphological dilate operation. These regions are then substituted by local noise and therefore do not appear in the remaining steps. Once all the bright sources are removed from the image, the SWT is applied until scale $J$ (as is illustrated in Figure 3, where $\mathrm{J}=6$ ). Note that the last images of this figure are the ones containing the information related to the extended low-intensity emitting objects. Hence, these images are automatically thresholded and the logical operator $O R$ is used to combine both images to obtain the final segmentation result.

As a way of summarizing our proposal, the following steps describe the whole algorithm:

1. The local noise in the original image is computed, deriving also a local threshold.

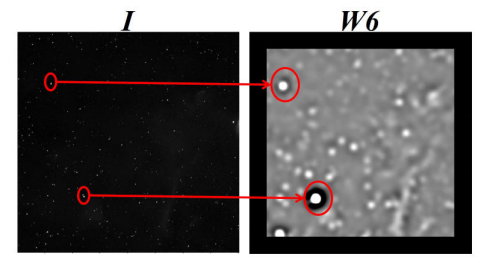

Fig. 4. Strong compact sources (as the ones circled in the original image on the left), show up in low spatial frequency planes (right).

2. Pixels with intensity levels over the threshold are labelled, and connected zones are then dilated in order to extract the bright sources.

3. Two different images are created: a "residual image" where bright sources have been substituted by local noise, and a binarized image with the bright sources.

4. A $J$-scale Stationary Wavelet decomposition using a $B_{3}$-spline filtering function is applied to the "residual image".

5. Local thresholding is applied to both the last scale and the smoothed array to perform the binarization.

6. Finally, both binarized images are combined using a logical operator $O R$ to produce the final extended emission segmentation of the image.

\section{RESULTS}

To illustrate the performance of our approach we used several Regions of Interest (ROIs) chosen from 14 of the mosaics obtained in the Phase I CGPS. All the chosen ROIs contained extended structures like SNRs or HII regions with surface temperatures much lower than the most bright sources in the region, which makes them suitable to validate our method. The mosaics used were A1, D1, F1, F2, IJ 2 and K2 of the CGPS at $1420 \mathrm{MHz}$. The ROIs were a portion of each mosaic of $260 \times 260,170 \times 150,200 \times 200,320 \times 260,296 \times 246$ and $240 \times 202$ pixels, respectively. All the images were 32 bpp. The scale $n$ used in the decomposition was $J=3$ or $J=4$ depending on the size of the image. In addition we also used the $1824 \times 1824$ image composition corresponding to the mosaics V1, V2, W1, W2, X1, X2, Y1 and Y2 (which corresponds to the areas shown in figures 1 to 4) to illustrate the performance of the method in large areas with a high density of faint extended emission. In this last case $J=6$.

Figure 5 shows the results of applying our approach to 6 different morphological structures with different intensities. The first column shows the original image, the second one shows the image with the contrast modified by an expert, 
while the last one shows the obtained results when applying our algorithm to the original images. Note that our approach automatically obtained the extended sources present in the data, but not visible without the manual expert enhancement. We want to remark here the importance of removing the bright sources and substitute them by local noise. This step clearly improves the final segmentation of the extended structures.

\section{CONCLUSIONS}

The segmentation of extended and irregular structures in radio-astronomical images is a challenging task due to the fact that they are found at different intensity scales, they are morphologically complex and irregular, and also to the overlap with compact and brighter sources. In this paper a new proposal for their automatic segmentation has been presented. The approach is built on a multi-resolution framework that allows to detect extended sources at different intensities and also to hide the strong sources which pollute the rest of the image. The results obtained from the experimental tests have shown that our approach is able to correctly detect such structures. Furthermore, the proposed segmentation process may be also applied directly to other low frequency wavelengths surveys (such infrared). Future work will be directed to the classification of these detected structures according to their morphology for further automatic catalogation.

\section{ACKNOWLEDGEMENTS}

This research used the facilities of the Canadian Astronomy Data Centre operated by the National Research Council of Canada with the support of the Canadian Space Agency. The research presented in this paper has used data from the Canadian Galactic Plane Survey a Canadian project with international partners supported by the Natural Sciences and Engineering Research Council.

\section{REFERENCES}

[1] A. R. Taylor, et al., "The canadian galactic plane survey," Astronomical Journal, vol. 125, pp. 3145, 2003.

[2] M. Peracaula, J. Freixenet, J. Martí, J. Martí, and J. M. Paredes, "Faint compact object detection in wide field interferometric radio images using a hybrid method based on local thresholding and wavelet decomposition.," in Astronomical Data Analysis Software and Systems XVII, 2008.

[3] A. Torrent, M. Peracaula, X. Lladó, J. Freixenet, J.R. Sánchez-Sutil, and J. Martí, "Detecting faint compact sources using local features and a boosting approach," in IAPR International Conference on Pattern Recognition, 2010, pp. 4613-4616.
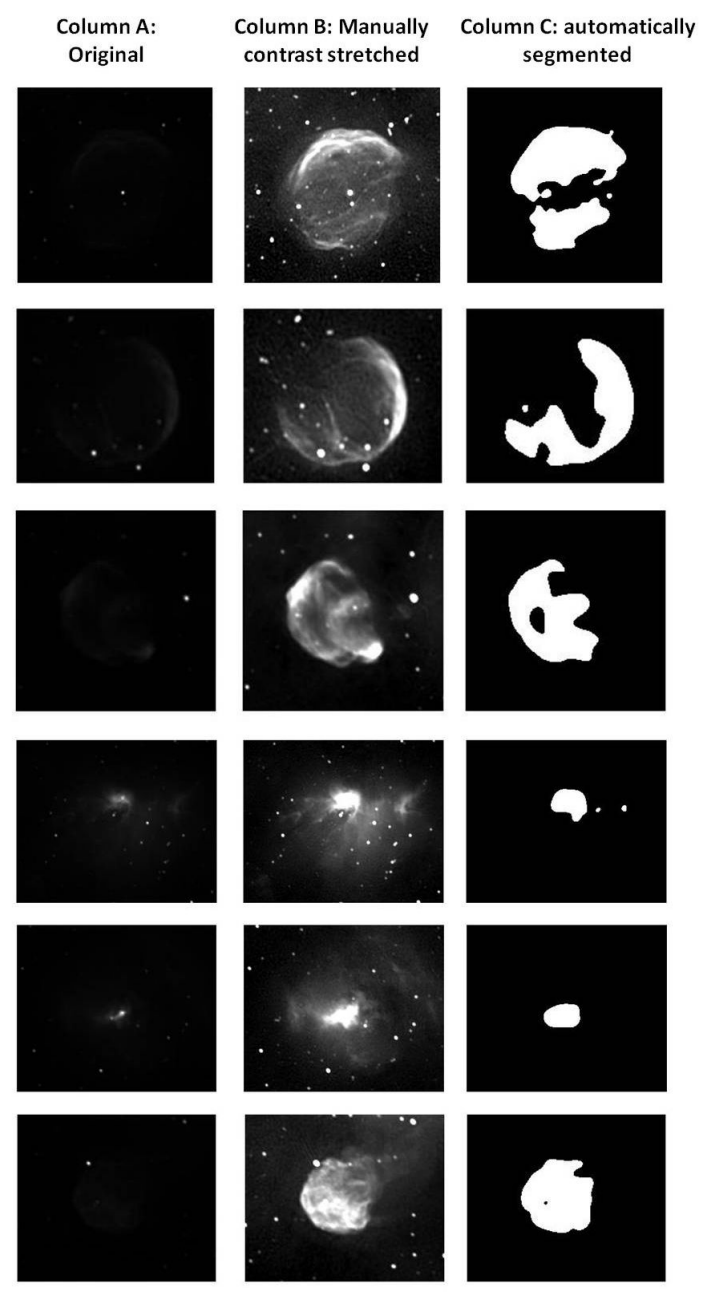

Fig. 5. Examples of segmentation results. First column shows the original image. Second column shows the image with the contrast modified by an expert. Last column shows the obtained segmentation results.

[4] Soo Chang Kim and Tae Jin Kang, “Texture classification and segmentation using wavelet packet frame and gaussian mixture model," Pattern Recognition, vol. 40, no. 4, pp. 1207-1221, 2007.

[5] A. Bijaoui and F. Rué, "A multiscale vision model," Signal Processing, vol. 46, pp. 345, 1995.

[6] J.L. Starck and F. Murtagh, Astronomical Image and Data Analysis, Springer-Verlag, New York, 2002.

[7] J.L. Starck and F. Murtagh, "Image restoration with noise suppression using the wavelet transform," Astronomy \& Astrophysics, vol. 288, no. 1, pp. 342-348, 1994. 